\title{
Efeitos do exercício físico sobre o perfil lipídico e inflamatório de mulheres jovens em uso de contraceptivo oral combinado: protocolo de um estudo sequencial cruzado
}

\author{
Effects of physical exercise on the lipid and \\ inflammatory profile of young women using \\ combined oral contraceptives: protocol of a cross- \\ sectional study
}

\begin{abstract}
RESUMO | INTRODUÇÃO: O uso de contraceptivos orais combinados (COC) trata-se de um fator de risco para a doença aterosclerótica por comprometer o perfil lipídico e inflamatório, podendo o exercício físico minimizar essas condições. OBJETIVO: Testar a hipótese de que exercício físico pode modificar o perfil lipídico e inflamatório de mulheres em uso COC. MÉTODOS: Protocolo de um estudo sequencial cruzado com mulheres de 20 e 30 anos, irregularmente ativas, em uso de COC há pelo menos 6 meses. Realizouse uma avaliação físico-clínica (AFC) nas participantes com medidas antropométricas, VO2máx. indireto e análise do perfil lipídico e inflamatório. Na sequência separou-se as participantes em 2 grupos: O grupo intervenção inicial (GII) que iniciou praticando exercícios intervalados de alta intensidade por 2 meses, e o grupo intervenção posterior (GIP), que seguiu irregularmente ativo pelo mesmo período. Em seguida o GIl e o GIP alternariam suas condições por mais 2 meses, totalizando 4 meses de acompanhamento e 3 AFC, realizadas no início, após 2 meses e ao final do estudo. As informações colhidas foram divididas em 3 momentos: Momento inicial (MI), momento pós exercício (MPE) e pós inatividade (MPI).
\end{abstract}

PALAVRAS-CHAVE: Contraceptivos orais combinados. Exercício físico.
Juliane Barbosa' 1 (1)

Vinícius Afonso Gomes ${ }^{2}$ (1)

Marvyn de Santana do Sacramento ${ }^{3}$ (1) Marco Antônio Ferreira 4 Jefferson Petto ${ }^{5}$ (1)

lica (Salvador). Bahia, Brasil. juul.barbosa@gmail.com ${ }^{2}$ Centro Universitário UniRuy (Salvador). Bahia, Brasil. vinifisioterapia@yahoo.com.br lica (Salvador). Bahia, Brasil. marvynsantana@gmail.com Faculdade do Centro Oeste Paulista (Bauru). São Paulo, Brasil. marcaumsf@gmail.com Cara Cardiovascular, Respiratória e Metabólica (Salvador), Escola Bahiana de Medicina e Saúde Pública (Salvador). Bahia, Brasil. gfpecba@bol.com.br

ABSTRACT | INTRODUCTION: The use of combined oral contraceptives $(\mathrm{COC})$ is a risk factor for atherosclerotic disease because it compromises the lipid and inflammatory profile, and physical exercise can minimize these conditions. OBJECTIVE: To test the hypothesis that high intensity interval physical exercise promotes changes in the lipid and inflammatory profile of women who are irregularly active using COC. METHODS: Protocol of a crosssectional study with women of 20 and 30 years, irregularly active, using $\operatorname{COC}$ for at least 6 months. A physicalclinical assessment (AFC) was performed on the participants with anthropometric measurements, VO2max. analysis and analysis of the lipid and inflammatory profile. Afterwards, the participants were divided into 2 groups: the initial intervention group (GII) that started practicing high intensity interval exercises for 2 months, and the posterior intervention group (GIP), which remained irregularly active for the same period. Then the GII and GIP would alternate their conditions for another 2 months, totaling 4 months of follow-up and 3 AFC, performed at the beginning, after 2 months and at the end of the study. The information collected was divided into 3 moments: Initial moment (MI), post-exercise moment (MPE) and post-inactivity (MPI).

KEYWORDS: Combined oral contraceptives. High intensity interval exercise. 


\section{Introdução}

Relatos históricos afirmam que mulheres em todo o planeta começaram a utilizar métodos contraceptivos rudimentares há pelo menos 4 mil anos. Com o passar do tempo, esses métodos foram se aperfeiçoando, até que entre os anos de 1950 e 1960 surgem os primeiros contraceptivos orais nos Estados Unidos da América ${ }^{1,2}$. Constituídos, em sua maioria, por um componente estrogênico e outro progestagênico, que em pouco tempo passaram a ser comercializados em todo o mundo devido a sua eficácia, baixo custo e viabilidade de utilização².

Dentre os possíveis efeitos colaterais do uso dos contraceptivos orais, podemos destacar a elevação de endotelinas, do peroxinitro, da angiotensina 2, do estresse oxidativo e da resistência insulínica 4,5 . Bem como também pode ocorrer a redução do oxido nítrico, da prostaciclina e alteração dos hepatócitos ${ }^{3-6}$.

Todas essas novas condições favorecem alterações trombolíticas, vasoconstrictoras, inflamatórias e lipídicas; que em combinação, podem promover uma série de injúrias cardiovasculares determinantes para a doença aterosclerótica $3,7, \frac{1}{8}$.

Em 2013, um estudo transversal 13 demonstrou que o perfil inflamatório avaliado pelos níveis de proteína C-reativa (PCR) apresentava-se significativamente maior em usuárias de contraceptivos orais combina$\operatorname{dos}(\mathrm{COC})$.

Um outro estudo pôde verificar uma menor magnitude da lipemia pós-prandial (LPP) em mulheres que não usavam $\mathrm{COC}$, ficando claro nesses dois casos que a terapia contraceptiva influência o perfil inflamatório e lipídico ${ }^{10}$.

Como meio de atenuar ou até mesmo reverter essas condições, o exercício físico tem sido amplamente empregado em diversas populações mundo afora ${ }^{11}$.

Foi com esse pensamento que Petto et al. ${ }^{\frac{12}{2}}$ avaliaram tanto a lipemia pós-prandial, quanto o perfil inflamatório de mulheres que se autodeclararam "irregularmente ativas" e "ativas" em uso de COC. Foi observado que as participantes do grupo "ativo" apresentaram menores níveis de inflamação subclínica, e que apesar de ambos os grupos estarem com níveis de triglicerídeos dentro da normalidade, o "grupo irregularmente ativo" manifestou valores
56\% maiores, estando dessa forma mais próximo do limiar de anormalidade ${ }^{12}$.

Sabe-se que o perfil inflamatório e lipídico é maior em usuárias de $\operatorname{COC}^{9,10,12}$, sobretudo naquelas que possuem menores níveis de atividade física ${ }^{12}$. Pensando nisso, o exercício físico tem sido empregado para atenuar ou até mesmo reverter essas condições em algumas populações ${ }^{11}$.

No entanto, em nossos estudos prévios, não foram observados estudos que verificaram a relação de causa-efeito do exercício físico em mulheres que fazem uso contínuo de COC.

\section{Objetivo}

Testar a hipótese de que exercício físico pode modificar o perfil lipídico e inflamatório de mulheres em uso de contraceptivos orais combinados.

\section{Hipóteses}

HO - Um programa de exercício físico intervalado de alta intensidade não reduz o perfil lipídico e inflamatório de mulheres jovens irregularmente ativas em uso de contraceptivo oral combinado.

H1 - Um programa de exercício físico intervalado de alta intensidade reduz o perfil lipídico e inflamatório de mulheres jovens irregularmente ativas em uso de contraceptivo oral combinado.

\section{Métodos}

\section{Desenho do estudo}

A pesquisa se caracteriza como protocolo de um estudo sequencial cruzado registrado no Registro Brasileiro de Ensaios Clínicos (ReBEC), com numeração RBR-4jm343.

\section{População do estudo}

Mulheres jovens normolipídicas irregularmente ativas em uso contínuo de contraceptivo oral combinado. 


\section{Critérios de inclusão}

Mulheres irregularmente ativas das categorias A e $B$, com idade entre 20 e 30 anos, nulíparas, em uso contínuo de COC há pelo menos 6 meses. Os COC a serem usados deveriam ser de quarta geração e ter uma dosagem de estrogênio entre 15 e 35 microgramas. Para comprovar o nível de atividade física e categoria das participantes do estudo, foi utilizado o International Physical Activity Questionnaire (IPAQ) ${ }^{18}$, na versão curta. Esse questionário foi utilizado, pois o mesmo apresenta as seguintes vantagens: pode ser realizado em duas formas (curta e longa), possibilita se estimar o gasto calórico e apresenta uma classificação mais detalhada em sedentário, irregularmente ativo, ativo e muito ativo, além de possibilitar maior chance de comparações e estar adaptado a realidade brasileira ${ }^{13}$.

\section{Critérios de exclusão}

Serão excluídas as mulheres que referirem e apresentarem condições físico-clínicas incompatíveis a realização de exercício físico como relatos de hipoglicemia, quadro álgico muscular ou articular de membros inferiores, hipotensão postural, labirintite, dislipidemias, disfunção hepática, diabetes/pré-diabetes, hipo ou hipertireoidismo, síndrome do ovário policístico, doenças renais, uso de suplementos alimentares ou anabolizantes e de dieta hipo ou hiperlipídica, histórico de alcoolismo, tabagismo, uso de hipolipemiantes, corticoides, diuréticos ou betabloqueadores e o índice de massa corporal (IMC) acima de $30 \mathrm{~kg} / \mathrm{m}^{2}$.

\section{Aspectos éticos}

Durante todo o estudo, serão observadas as diretrizes sobre a pesquisa com seres humanos da Declaração de Helsinque e da Resolução 466/12 do Conselho Nacional de Saúde. Este estudo foi submetido e aprovado pelo Comitê de Ética em Pesquisa da Faculdade Nobre de Feira de Santana - CAAE: 79549517.3.0000.5654. Todas as participantes receberão detalhadamente as informações sobre os objetivos do estudo, riscos e benefícios envolvidos nos procedimentos e assinarão o termo de consentimento livre e esclarecido (TCL). Serão preenchidas duas vias, ficando uma em posse das participantes e a outra com os pesquisadores.

\section{Calculo amostral}

Para cálculo amostral, foi realizado um estudo piloto prévio $(n=3)$, considerando como variável desfecho triglicerídeos com Média e DP = $32 \pm 9$, sendo necessárias 22 voluntárias. Foi considerando um alfa $=0,05$ (bidirecional) e beta $=0,80$; adotando como significativa uma diferença de $20 \%$ para a variável triglicerídeos entre os momentos. Tendo em vista que o coeficiente de variação laboratorial da dosagem dos triglicerídeos é de $5 \%$, e que uma diferença quatro vezes maior que a esperada anula o viés desse coeficiente de variação analítica, será feito um cruzamento, portanto serão necessárias 12 participantes, com 6 alocadas para o GIl e outras 6 alocadas para o GIP. O cálculo amostral foi realizado no WinPepi, versão 11.65.

\section{Coleta de dados}

Para coletar informações gerais sobre as características da amostra, todas as participantes do estudo que forem selecionadas serão submetidas a uma Avaliação Físico Clínica (AFC) que será baseada em 4 etapas.

1 = Aplicação de um questionário padrão: Com o objetivo de realizar uma triagem da amostra a respeito de informações relevantes ao protocolo de estudo em um dado momento;

2 = Avaliação dos sinais vitais/exame físico: Composto por medidas de frequência cardíaca e pressão arterial em repouso, massa corporal total, estatura e circunferência abdominal. Para mensuração da frequência cardíaca, será utilizado cardiofrequencímetro de pulso da marca Polar®. Para aferição da pressão arterial, serão seguidas as recomendações da Sociedade Brasileira de Hipertensão ${ }^{13}$, será utilizado um esfigmomanômetro e estetoscópio das marcas WelchAllyn ${ }^{\circledR}$ e Littman ${ }^{\circledR}$, respectivamente. A estatura será medida com auxílio de estadiômetro profissional Sanny ${ }^{\circledR}$ com precisão de $0,1 \mathrm{~cm}$, executada com as participantes descalças, com os glúteos e ombros apoiados em encosto vertical. A massa corporal total será obtida com balança digital Filizola ${ }^{\circledR}$ de capacidade máxima de $150 \mathrm{~kg}$, aferida pelo Inmetro, com certificado próprio especificando margem de erro de $\pm 100 \mathrm{~g}$. A circunferência abdominal será obtida com fita métrica metálica e inelástica, marca Starrett, com definição de medida de 0,1 cm. Será mensurada na menor curvatura localizada entre 
a última costela e a crista ilíaca sem comprimir os tecidos $^{14}$. O índice de massa corporal (IMC) será calculado com as medidas de massa e altura, de acordo com a equação de Quetelet: IMC = massa $(\mathrm{kg}) /$ altura2 $(\mathrm{m})$. Os pontos de corte adotados serão os preconizados pela IV Diretriz Brasileira sobre Dislipidemias e Prevenção da Aterosclerose do Departamento de Aterosclerose da Sociedade Brasileira de Cardiologia ${ }^{15}$, ou seja, baixo peso (IMC $<18,5)$, eutrofia $(18,5<$ IMC $<24,9)$, sobrepeso $(25<$ IMC $<29,9)$ e obesidade $(I M C \geq 30)$.

3 = Teste de graduação do consumo máximo de oxigênio (VO2 máx.) indireto por meio do protocolo de Cooper, realizado na esteira ${ }^{19}$ : Nesse teste, as participantes inicialmente serão orientadas a respeito de todas as etapas do teste e posteriormente orientadas a realizar um aquecimento feito sob a forma de caminhada em velocidade que represente uma auto percepção de esforço nível "fácil", regulada pela própria pessoa avaliada durante 5 minutos em uma esteira ergométrica sem inclinação da marca Movement ${ }^{\circledR}$. Imediatamente após o período de aquecimento a esteira será desligada e instantaneamente religada, sendo que a partir desse momento a avaliação será de fato iniciada, e a participante deverá percorrer em ritmo e regulação próprios a maior distância possível, será permitido correr, marchar e caminhar. Após o $12^{\circ}$ minuto de avaliação, a distância percorrida será visualizada no hodômetro da esteira e registrada na ficha da participante, sendo finalizada nesse instante, com posterior desaquecimento da esteira, com redução gradual da velocidade que será realizada em 2 minutos até que a velocidade da esteira esteja zerada. Se por algum eventual motivo for necessário zerar a velocidade da esteira durante os 12 minutos de teste, o mesmo deverá ser interrompido e cancelado naquele dia, com nova realização após 72 horas.

4 = Coleta sanguínea: Após o período mínimo de $72 \mathrm{~h}$ do teste de Cooper, as participantes do estudo serão submetidas a uma coleta de amostra sanguínea para VPLI no período matutino, com $12 \mathrm{~h}$ e jejum, entre o quinto e o décimo dia do ciclo menstrual, considerando as menores flutuações hormonais, e/ou no $28^{\circ}$ dia sem medicação (fase inativa) conforme recomendado por Casazza et al. $\frac{16}{}$, para que não tenha influência do período menstrual no valor das variáveis sanguíneas analisadas. A VPLI será no Laboratório de Patologia Clínica (LPC) da unidade Barra, na cidade de Salvador$\mathrm{BA}$, que disponibilizará o espaço, materiais físicos e recursos humanos necessários para as coletas e análise laboratorial. Serão observados os valores de Colesterol total (CT); Triglicerídeos; (LDL)-colesterol; (HDL)-colesterol, (VLDL)-colesterol e PCR de alta sensibilidade; para que, de acordo com esses valores, sejam traçados os perfis lipídico e inflamatório da amostra. As participantes do estudo serão orientadas a não alterarem sua dieta na semana do teste e a não praticarem nenhum esforço físico diferente do habitual, bem como a não ingerirem bebidas alcoólicas nas $24 \mathrm{~h}$ precedentes ao teste. A avaliação do colesterol total, (HDL)-colesterol e triglicerídeos, será feita por método enzimático. O (LDL)-colesterol será calculado pela fórmula de Friedwald ${ }^{17}$ e o colesterol não HDL-C será calculado pela diferença entre colesterol total e (HDL)-colesterol. A PCR de AltaSensibilidade será mensurada por turbidimetria.

\section{Protocolo de Intervenção}

As voluntárias que serão destinadas ao momento de exercício físico passarão por uma avaliação dos sinais vitais no início, durante e ao final de cada sessão. Caso sejam conferidos padrões esperados para idade e nível de esforço, serão prosseguidas as atividades. 0 protocolo consiste em um treino intervalado de alta intensidade feito por meio de sprints, realizado em esteira ergométrica sem inclinação, com frequência de 2 vezes por semana e período total de 2 meses, como já descrito previamente. Durante as sessões de exercício a fase de aquecimento tem duração de 5 minutos com intensidade de $60 \%$ da frequência cardíaca de reserva predita (FCRP), que será calculada de acordo com a seguinte fórmula: $\{[(220$ - idade) - FC de repouso] $x 0,6]+F C$ de repouso\}. Na sequência, para a fase de condicionamento, será elevada a velocidade da esteira até que se alcançasse $90 \%$ da FCRP $\{[(220$ - idade $)$ - FC de repouso] $\times 0,9]+F C$ de repouso $\}^{20}$, mantendo essa velocidade por 1 minuto com posterior redução para velocidade de aquecimento pelos próximos 2 minutos, configurando o descanso ativo. Serão alternados os sprints aos momentos de descanso ativo por 10 vezes, com durações respectivas de 1 e 2 minutos (1' sprint e 2 ' descanso ativo), sendo que as 9 últimas velocidades dos sprints e dos descansos ativos serão mantidas de acordo com as velocidades alcançadas na primeira fase de cada um desses momentos. Para a fase de desaquecimento ao final da sessão, será mantida a velocidade idêntica à velocidade de aquecimento, com duração de 2 minutos, até que a esteira seja desligada conforme podemos observar na figura 1 . 


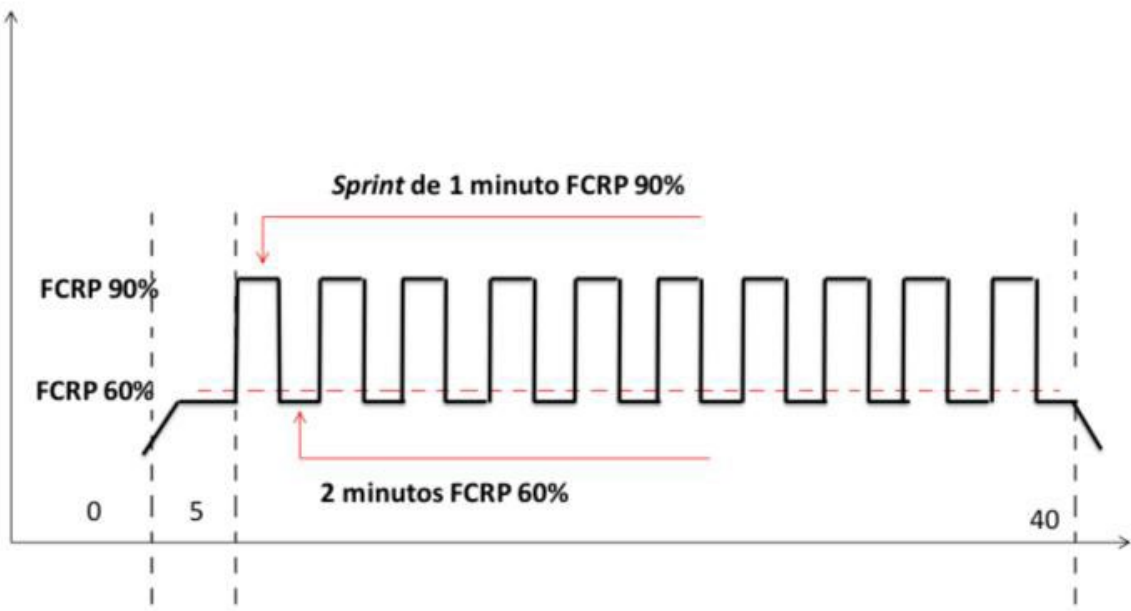

Treino baseado de acordo com a frequência cardíaca de reserva predita (FCRP). Os sprints terão duração de 1 minuto e velocidades referentes ao valor alcançado no primeiro sprint. Os períodos de descanso ativo terão duração de 2 minutos e velocidade referente a alcançada no aquecimento da sessão.

As participantes serão monitorizadas pelo mesmo cardiofrequencímetro de pulso da marca Polar®, utilizado durante o protocolo de Cooper que mensura o gasto calórico da sessão, baseado no IMC, na idade, na FC máxima prevista e na média da FC durante o exercício 19 .

\section{Período de acompanhamento}

O protocolo de intervenção, bem como os três primeiros passos descritos na avaliação físico-clínica, acontecerá na Fisiocordis Clínica de Reabilitação Cardiovascular, localizada na cidade de Salvador-BA, que também disponibilizará seu espaço, materiais físicos e recursos humanos. Logo após o primeiro teste de VPLI, aquelas seis primeiras participantes ingressantes no estudo serão alocadas para o GII, e as seis últimas serão direcionadas para o grupo de intervenção posterior GIP. A princípio, somente as participantes do (GII) ingressarão no programa de exercício físico intervalado de alta intensidade. Aquelas participantes do (GIP) vão se manter com o mesmo nível de atividades físicas de antes do início do estudo, com período de acompanhamento de 2 meses para cada grupo. Uma segunda AFC será então realizada após esse período, seguida de alternância dos grupos em relação ao exercício físico e a inatividade física em mais outros 2 meses. Por fim, uma terceira e última AFC será realizada, totalizando um follow-up de aproximadamente 4 meses, conforme observado na figura 2.

Figura 2. Desenho do estudo

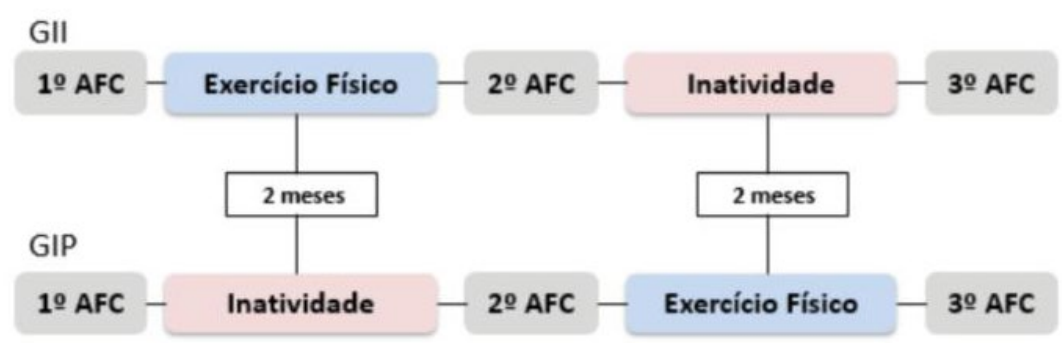

Desenho do protocolo de um estudo sequencial cruzado, onde ambos os grupos passarão por 3 avaliações físico-clínicas (AFC), realizadas no início do estudo, após 2 e 4 meses de seguimento. 0 Grupo intervenção inicial (GII) realizará o protocolo de exercício intervalado de alta intensidade logo após a $1^{\text {a }} \mathrm{AFC}$, sendo que após a $2^{\mathrm{a}} \mathrm{AFC}$, será iniciado o período de inatividade, seguido pela

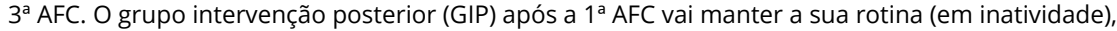
após 2 meses realizará nova AFC e iniciará um período de 2 meses de exercício físico intervalado de alta intensidade, sendo que no final desse período será realizada a última AFC. 
Para verificação da distribuição dos dados serão aplicados os testes de simetria e curtose, o teste de Shapiro-Wilk, além da inspeção visual dos histogramas. Nas variáveis que apresentarem distribuição não-normal, será utilizado medida de tendência central e dispersão a mediana e desvio interquartil, respectivamente, com utilização do teste de Kruskal Wallis para medida de comparação. Para todas as demais variáveis, será utilizado a média e o desvio padrão verificados por meio do teste Anova, com medidas repetidas e pós-teste de Tukey. Todas as análises serão realizadas no pacote estatístico SPSS (Statistical Package for the Social Sciences), versão 21.0, adotando-se um nível de significância de $5 \%$.

Os resultados colhidos do grupo GII e GIP serão distribuídos em 3 momentos distintos:

1 - Momento inicial (MI), 2 - momento pós exercício (MPE) e 3 - momento pós inatividade (MPI).

Para avaliação dos dados do "momento inicial" do grupo GIl e GIP serão combinados, obtendo-se os valores de tendência central e dispersão resultante de 12 coletas. Um cruzamento será feito entre os dados dos MPE e MPI do GII e GIP, com a diferença de que no Gll os dados do MPE serão colhidos 2 meses após o início do estudo, ao passo que no GIP esses mesmos dados só poderiam ser coletados após 4 meses. Da mesma forma, os dados do MPI já poderão ser coletados no GIP após 2 meses do início do estudo, só sendo passíveis de coleta após 4 meses no Gll. Após todos os cruzamentos de dados, as comparações das variáveis investigadas serão realizadas entre os três momentos distintos da seguinte forma: MI vs. MPE; MPE vs. MPI e MI vs. MPI. Esse cruzamento tem como objetivo fazer com que as participantes sejam controles de si mesmas, minimizando os riscos de vieses.

\section{Variáveis}

Variável preditora - Exercício físico intervalado de alta intensidade.

Variável desfecho - Perfil lipídico e inflamatório.

Variável confundidora - Alimentação e estilo de vida.
Ao financiamento da Coordenação de Aperfeiçoamento de Pessoal de Nível Superior (CAPES).

\section{Contribuições dos autores}

Gomes AF participou da concepção e redação do manuscrito. Barbosa J, Sacramento MS e Ferreira MA participaram da redação do manuscrito. Petto J participou da concepção, redação do manuscrito e revisão crítica do manuscrito quanto ao conteúdo intelectual.

\section{Conflitos de interesses}

Nenhum conflito financeiro, legal ou político envolvendo terceiros (governo, empresas e fundações privadas, etc.) foi declarado para nenhum aspecto do trabalho submetido (incluindo, mas não se limitando a subvenções e financiamentos, participação em conselho consultivo, desenho de estudo, preparação de manuscrito, análise estatística, etc.).

\section{Referências}

1. Colquitt CW, Martin TS. Contraceptive Methods: A Review of Nonbarrier and Barrier Products. J Pharm Pract. 2017;30(1):130-5. https://doi.org/10.1177\%2F0897190015585751

2. Watkins ES. On the Pill, A Social History of Oral Contraceptives, 1950- 1970. Baltimore: Johns Hopkins University Press; 1998.

3. Gevaert AB, Lemmens K, Vrints CJ, Craenenbroeck EM. Targeting Endothelial Function to Treat Heart Failure with Preserved Ejection Fraction: The Promise of Exercise Training. Oxid Med Cell Longev. 2017;2017:4865756. https://doi. org/10.1155/2017/4865756

4. Beck P. Effect of Progestins on Glucose and Lipid Metabolism. Ann N Y Acad Sci. 1977;286(1):434-45. https://doi. org/10.1111/j.1749-6632.1977.tb29435.x

5. Cauci S, Di Santolo M, Culhane JF, Stel G, Gonano F, Guaschino $\mathrm{S}$. Effects of thirdgeneration oral contraceptives on highsensitivity C-reactive protein and homocysteine in young women. Obstet Gynecol. 2008;111(4):857-64. https://doi.org/10.1097/ aog.0b013e31816a2476

6. Silvestri A, Gebara O, Vitale C, Wajngarten M, Leonardo F, Ramires JA et al. Increased levels of C-reactive protein after oral hormone replacement therapy may not be related to an increased inflammatory response. Circulation. 2003;107(25):3165-9. https:// doi.org/10.1161/01.cir.0000074208.02226.5e

\section{Desfechos primários}

Perfil lipídico e inflamatório. 
7. Signori LU, Plentz RDM, Irigoyen MC, Schaan BD. O papel da lipemia pós-prandial na gênese da aterosclerose: particularidades do diabetes mellitus. Arq Bras Endocrinol Metab. 2007;51(2):22231. https://doi.org/10.1590/S0004-27302007000200011

8. Gupta A, Baradaran H, Al-Dasuqi K, Knight-Greenfield A, Giambrone AE, Delgado D, et al. Gadolinium Enhancement in Intracranial Atherosclerotic Plaque and Ischemic Stroke: A Systematic Review and Meta-Analysis. J Am Heart Assoc. 2016;5(8):e003816. https://doi.org/10.1161/jaha.116.003816

9. Petto J, Pereira LS, Santos ACN, Giesta BA, Melo TA, Ladeia AMT. Inflamação subclínica em mulheres que utilizam contraceptivo oral. Rev Bras Cardiol [Internet]. 2013;26(6):465-71. Disponível em: http://www.onlineijcs.org/english/sumario/26/pdf/v26n6a08.pdf

10. Petto J, Vasques LM, Pinheiro RL, Giesta BA, Santos AC, Gomes Neto $\mathrm{M}$, et al. Comparação da Lipemia Pós-Prandial de Mulheres que Utilizam e Não Utilizam Contraceptivo Oral. Arq Bras Cardiol. 2014;103(3):245-50. http://dx.doi.org/10.5935/abc.20140080

11. Rubio Pérez FJ, Franco Bonafonte L, Ibarretxe Guerediaga D, Oyon Belaza MP, Ugarte Peyron P. Effect of an individualised physical exercise program on lipid profile in sedentary patients with cardiovascular risk factors. Clin Investig Arterioscler. 2017;29(5):201-8. https://doi.org/10.1016/j.arteri.2017.02.002

12. Petto J, Silveira DW, Santos AC, Seixas CR, Santo DG, Oliveira FT, et al. Lipemia pós-prandial e inflamação subclínica em mulheres ativas que utilizam contraceptivo oral. Int J Cardiovasc Sci. 2015;28(3):215-23. http://www.dx.doi.org/10.5935/23594802.20150031

13. Sociedade Brasileira de Cardiologia SB de HSB de N. VI Diretrizes brasileiras de hipertensão. Arq Bras Cardiol [Internet]. 2010;95(supl. 1):1-51. Disponível em: http://publicacoes.cardiol. br/consenso/2010/Diretriz hipertensao associados.pdf
14. WHO Consultation on Obesity. Obesity: preventing and manging the global epidemic - report of a WHO consultation [Internet]. Geneva (Suíça): World Health Organization; 2000. Disponível em: https://apps.who.int/iris/handle/10665/42330

15. Sposito AC, Caramelli B, Fonseca FA, Bertolami MC, Afiune Neto A, Souza AD, et al. IV Diretriz Brasileira sobre Dislipidemias e Prevenção da Aterosclerose: Departamento de Aterosclerose da Sociedade Brasileira de Cardiologia. Arq. Bras. Cardiol. 2007;88(suppl 1):2-19. https://doi.org/10.1590/S0066782X2007000700002

16. Casazza GA, Suh SH, Miller BF, Navazio FM, Brooks GA. Effects of oral contraceptives on peak exercise capacity. J Appl Physiol (1985). 2002;93(5):1698-702. https://doi.org/10.1152/ japplphysiol.00622.2002

17. Friedewald WT, Levy RI, Fredrickson DS. Estimation of the concentration of low-density lipoprotein cholesterol in plasma, without use of the preparative ultracentrifuge. Clin Chem. 1972;18(6):499-502. Citado em: PMID: 4337382

18. Hagströmer M, Oja P, Sjöström M. The International Physical Activity Questionnaire (IPAQ): a study of concurrent and construct validity. Public Health Nutr. 2006;9(6):755-62. https://doi. org/10.1079/phn2005898

19. Cooper $\mathrm{KH}$. Correlations between field and treadmill testing as a means for assessing maximal oxygen intake. JAMA. 1968: 203(3):201-4. Cited em: PMID: 5694044

20. Negrão CE, Barretto ACP. Cardiologia do exercício: do atleta ao cardiopata. 3rd. ed. Barueri (SP): Editora Manole; 2010. 